\title{
Electrostriction enhancement in metamaterials
}

\author{
M. J. A. Smith, ${ }^{1,2,{ }^{*}}$ B. T. Kuhlmey, ${ }^{1,2}$ C. Martijn de Sterke, ${ }^{1,2}$ C. Wolff, ${ }^{3}$ M. Lapine, ${ }^{3}$ and C. G. Poulton ${ }^{3}$ \\ ${ }^{1}$ Centre for Ultrahigh bandwidth Devices for Optical Systems (CUDOS), School of Physics, The University of Sydney, NSW 2006, Australia \\ ${ }^{2}$ Institute of Photonics and Optical Science (IPOS), School of Physics, The University of Sydney, NSW 2006, Australia \\ ${ }^{3}$ Centre for Ultrahigh bandwidth Devices for Optical Systems (CUDOS), School of Mathematical and Physical Sciences, \\ University of Technology Sydney, NSW 2007, Australia
}

(Received 19 April 2015; revised manuscript received 8 May 2015; published 4 June 2015)

\begin{abstract}
We demonstrate a controllable enhancement in the electrostrictive properties of a medium using dilute composite artificial materials. Analytical expressions for the composite electrostriction are derived and used to show that enhancement, tunability, and suppression can be achieved through a careful choice of constituent materials. Numerical examples with $\mathrm{Ag}, \mathrm{As}_{2} \mathrm{~S}_{3}, \mathrm{Si}$, and $\mathrm{SiO}_{2}$ demonstrate that even in a nonresonant regime, artificial materials can bring more than a threefold enhancement in the electrostriction.
\end{abstract}

DOI: 10.1103/PhysRevB.91.214102

PACS number(s): 77.65.Bn, 78.20.H-, 81.05.Xj, 42.65.Es

\section{INTRODUCTION}

Optoacoustic interactions have gained considerable attention in recent years in the context of nanophotonics [1]. One of the strongest and most important of these is stimulated Brillouin scattering (SBS) [2-4], which is a coherent interaction between the electromagnetic and acoustic fields occurring in an optical waveguide. SBS has been demonstrated in a number of areas within nanophotonics, notably in the design of nanoscale devices for Brillouin lasers, signal processing, and microwave generation [1]. The strength of SBS is principally determined by the electrostriction, which is the induced strain arising from an electromagnetic field within the waveguiding material. The magnitude of the electrostrictive effect, as well as that of the related photoelastic effect, has widely been considered a property of the material used, and as a consequence, the materials that have been used in SBS studies have been mostly limited to those with naturally large electrostriction constants.

At the same time, it is well established in the metamaterials literature that large enhancements in the nonlinear properties of a medium can be achieved through the use of composites that have subwavelength structural features [5]. Metamaterials have been used to enhance nonlinear scattering effects such as the Raman effect [6], to achieve nonlinear diffraction [7], and have been used in optomechanical systems [8] at microwave frequencies [9]. However, nonlinear metamaterials have yet to be designed for the enhancement and suppression of electrostriction and photoelasticity, particularly in the optical range.

In this paper, we demonstrate that artificial materials can be designed for the tunable enhancement or suppression of electrostriction. We investigate materials consisting of a dilute suspension of spheres embedded in a dielectric matrix, as presented in Fig. 1. We consider both dielectric and metallic inclusions, and derive a mixing formula that describes the effective electrostriction of the composite. The electrostriction for a selection of practically realizable examples is then evaluated, and used to show that enhancement or suppression of electrostriction can be achieved. To our knowledge, we are the first to explore modifications in the optoacoustic material

\footnotetext{
*m.smith@physics.usyd.edu.au
}

properties of a medium. It has been shown previously that even very simple composite material designs can enhance the nonlinear susceptibility beyond that of either constituent materials [10] and, therefore, we expect similar enhancements here with the electrostriction.

To determine the electrostrictive properties of a composite material, we must first obtain electrostriction values for all constituent media. Expressions for these constituents can differ depending on whether dispersion and loss are incorporated in their derivation, and less obviously, on other mechanical and thermodynamic assumptions that are imposed [11-14]. These considerations play an important part in determining regimes over which estimates for the electrostriction are appropriate, and a discussion of these relevant approximations can be found in the context of their derivations in the following.

The outline of this paper is as follows. In Sec. II, we derive a general expression for the electrostriction, including the effects of dispersion, and apply this to uniform dielectrics and metals. In Sec. III, we obtain the electrostriction for composite materials. In Sec. IV, we consider a series of practical examples before concluding remarks in Sec. V.

\section{ELECTROSTRICTION FOR CONSTITUENT MEDIA}

In this section, we derive a general expression for the electrostriction of a homogeneous material. Typically, estimates for the electrostriction of materials are made under the assumption of zero loss and dispersion, zero shear stress, and that variations in the permittivity arise from changes in density alone (i.e., an isothermal process) $[11,13,15]$. In a generalization of the standard procedure, we incorporate the effects of dispersion in our derivation. We begin by considering the electromagnetic energy density [13]

$$
u=\frac{1}{2} \varepsilon_{0} \frac{\partial\left(\omega \varepsilon_{\mathrm{r}}\right)}{\partial \omega}|\mathbf{E}|^{2},
$$

where $\varepsilon_{0}$ denotes the free-space permittivity, $\varepsilon_{\mathrm{r}}$ is the relative permittivity of the material, $\omega$ is the frequency, and $\mathbf{E}$ is the electric field. The change in energy density corresponding to a small change in the density $\rho$ is therefore

$$
\Delta u=\frac{1}{2} \varepsilon_{0} \frac{\partial}{\partial \rho}\left[\frac{\partial\left(\omega \varepsilon_{\mathrm{r}}\right)}{\partial \omega}\right]|\mathbf{E}|^{2} \Delta \rho,
$$




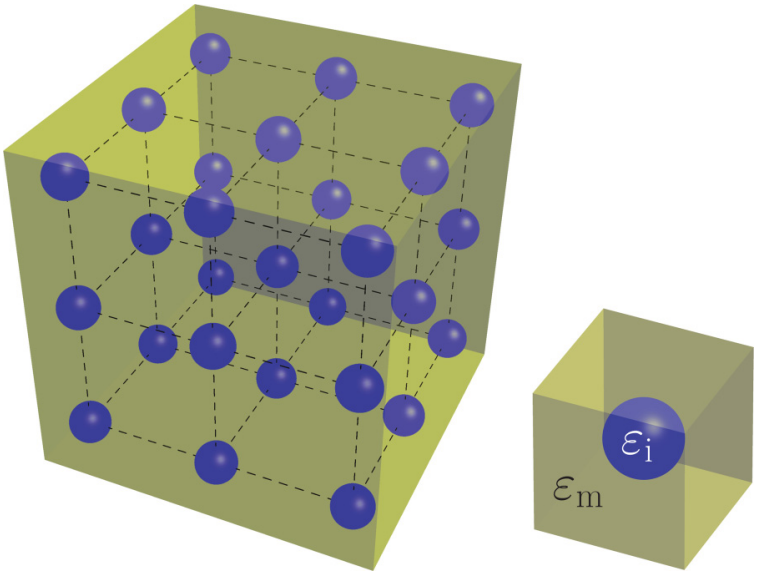

FIG. 1. (Color online) Schematic view of the metamaterial geometry investigated; a primitive cubic array of spheres in a host medium. Inset: fundamental unit cell for a cubic lattice of spheres.

where $\Delta$ denotes an infinitesimal quantity. Assuming an isothermal process, this change in the internal energy can be equated to the work done $W$ per unit volume $V$ by

$$
\Delta W=P \frac{\Delta V}{V}=-P \frac{\Delta \rho}{\rho},
$$

where $P$ is the induced hydrostatic pressure, to obtain

$$
P=-\frac{1}{2} \varepsilon_{0} \gamma|\mathbf{E}|^{2},
$$

and we define the electrostriction parameter

$$
\gamma=\rho \frac{\partial^{2}\left(\varepsilon_{\mathrm{r}} \omega\right)}{\partial \rho \partial \omega}
$$

as a nondimensional measure of the induced electrostrictive stress. From a microscopic perspective, the pressure field in Eq. (3) can be understood as arising from ionic movements in the material lattice induced by a Lorentz force $[16,17]$. From (4), we obtain expressions for the electrostriction of both dielectric and metallic media which are used in our composite model shown later.

\section{A. Electrostriction for dielectric media}

For a dielectric medium that is nondispersive and lossless, the electrostriction parameter (4) simplifies to the familiar form [11]

$$
\gamma=\rho \frac{\partial \varepsilon_{\mathrm{r}}}{\partial \rho}
$$

It is then usual to express this in terms of other wellknown material response tensors for practical evaluation. For example, for isotropic and homogeneous materials, (5a) is given by

$$
\gamma=\frac{1}{3} \varepsilon_{\mathrm{r}}^{2}\left(p_{11}+2 p_{12}\right),
$$

where $p_{i j}$ denotes the elasto-optic coefficients of the medium $[13,14,18]$. These $p_{i j}$ coefficients are well tabulated for a range of materials, and a selection of values for dielectric solids are presented in Table I for reference. However, to our knowledge no experimental data have been published on elasto-optic
TABLE I. Material parameters for a selection of dielectric materials at specified wavelengths $\gamma$ from Eq. (5b).

\begin{tabular}{lcccccc}
\hline Material & $\lambda(\mathrm{nm})$ & $\varepsilon_{\mathrm{r}}$ & $p_{11}$ & $p_{12}$ & $\gamma$ & Ref. \\
\hline $\mathrm{SiO}_{2}$ & 663 & 2.12 & 0.12 & 0.27 & 1.00 & {$[20]$} \\
$\mathrm{As}_{2} \mathrm{~S}_{3}$ & 1150 & 6.06 & 0.31 & 0.30 & 11.1 & {$[20]$} \\
$\mathrm{Si}$ & 3390 & 11.8 & -0.09 & 0.02 & -2.77 & {$[21]$} \\
\hline \hline
\end{tabular}

coefficients for metallic media [19], and so we now consider an estimate for the $\gamma$ of metals.

\section{B. Electrostriction for metallic media}

For metals, we return to (4), and use a simplified Drude model for the permittivity [22]

$$
\varepsilon_{\mathrm{r}}=1-\frac{\omega_{\mathrm{p}}^{2}}{\omega^{2}}
$$

to obtain a form for $\gamma$ which is useful for practical evaluation. Here, $\omega_{\mathrm{p}}^{2}=q^{2} N /\left(\varepsilon_{0} m_{\mathrm{e}}\right)$ is the square of the plasma frequency, $q$ is the electric charge, $m_{\mathrm{e}}$ is the effective mass of a constituent electron, $N=\rho / m$ is the number density, and $m$ is the mass density of the metal. Subsequently (4) and (6) give the estimate

$$
\gamma^{\mathrm{DM}}=\frac{\omega_{p}^{2}}{\omega^{2}} .
$$

We note that in the derivation of the metallic $\gamma$ above, we have neglected dissipation effects, which is consistent with the isothermal assumption made in the derivation of (4). The validity of this assumption is considered in the results section, with a discussion of attenuation.

Having derived evaluable expressions for dielectrics and metals, we now proceed to the electrostriction of composite materials.

\section{ELECTROSTRICTION FOR COMPOSITE MATERIALS}

In this section, we derive $\gamma$ for our composite material using the Maxwell-Garnett (MG) model. The effective permittivity given by this model is valid for a dilute array of spheres embedded in a host material, and has the form [23]

$$
\varepsilon_{\mathrm{r}}=\varepsilon_{\mathrm{m}}+\frac{3 \varepsilon_{\mathrm{m}}\left(\varepsilon_{\mathrm{i}}-\varepsilon_{\mathrm{m}}\right) f}{\left(\varepsilon_{\mathrm{i}}+2 \varepsilon_{\mathrm{m}}\right)-\left(\varepsilon_{\mathrm{i}}-\varepsilon_{\mathrm{m}}\right) f} .
$$

Here, $\varepsilon_{\mathrm{i}, \mathrm{m}}$ denotes the relative permittivities of the constituent materials, and we define the filling fraction

$$
f=\frac{V_{\mathrm{i}}}{V_{\mathrm{i}}+V_{\mathrm{m}}},
$$

where $V_{\mathrm{i}, \mathrm{m}}$ represent corresponding volumes. The subscript $\mathrm{i}$ denotes the inclusion and $\mathrm{m}$ denotes the matrix (constrained by the boundaries of the unit cell), as shown by the fundamental cell in Fig. 1. The only additional condition for (8) in our analysis is that the periodic array must be suitably subwavelength. An established rule of thumb is that the period of the lattice must be at least 10 times smaller than the optical wavelength in the material. To demonstrate this in a practical context, a wavelength in the material of $1 \mu \mathrm{m}$ and a filling 
fraction of $f=15 \%$ would correspond to an array period of $100 \mathrm{~nm}$ and a spherical radius of $33 \mathrm{~nm}$.

To begin, we consider a fully nondispersive model for the composite electrostriction.

\section{A. Nondispersive model}

Under the assumption that all constituent materials are nondispersive, the expression (4) reduces to the form given in Eq. (5a). Consequently, from the MG model we write

$$
\gamma^{\mathrm{ND}}=\rho\left[\frac{\partial \varepsilon_{\mathrm{r}}}{\partial \varepsilon_{\mathrm{m}}} \frac{\partial \varepsilon_{\mathrm{m}}}{\partial \rho}+\frac{\partial \varepsilon_{\mathrm{r}}}{\partial \varepsilon_{\mathrm{i}}} \frac{\partial \varepsilon_{\mathrm{i}}}{\partial \rho}+\frac{\partial \varepsilon_{\mathrm{r}}}{\partial f} \frac{\partial f}{\partial \rho}\right],
$$

where from (8) we have the partial derivatives

$$
\begin{aligned}
\frac{\partial \varepsilon_{\mathrm{r}}}{\partial \varepsilon_{\mathrm{m}}} & =\frac{\left[\left(\varepsilon_{\mathrm{i}}+2 \varepsilon_{\mathrm{m}}\right)^{2}+2 f\left(\varepsilon_{\mathrm{i}}-\varepsilon_{\mathrm{m}}\right)^{2}\right](1-f)}{\left[\left(\varepsilon_{\mathrm{i}}+2 \varepsilon_{\mathrm{m}}\right)-\left(\varepsilon_{\mathrm{i}}-\varepsilon_{\mathrm{m}}\right) f\right]^{2}}, \\
\frac{\partial \varepsilon_{\mathrm{r}}}{\partial \varepsilon_{\mathrm{i}}} & =\frac{\left[\left(\varepsilon_{\mathrm{i}}+2 \varepsilon_{\mathrm{m}}\right)-\left(\varepsilon_{\mathrm{i}}-\varepsilon_{\mathrm{m}}\right)\right]^{2} f}{\left[\left(\varepsilon_{\mathrm{i}}+2 \varepsilon_{\mathrm{m}}\right)-\left(\varepsilon_{\mathrm{i}}-\varepsilon_{\mathrm{m}}\right) f\right]^{2}}, \\
\frac{\partial \varepsilon_{\mathrm{r}}}{\partial f} & =\frac{3 \varepsilon_{\mathrm{m}}\left(\varepsilon_{\mathrm{i}}-\varepsilon_{\mathrm{m}}\right)\left(\varepsilon_{\mathrm{i}}+2 \varepsilon_{\mathrm{m}}\right)}{\left[\left(\varepsilon_{\mathrm{i}}+2 \varepsilon_{\mathrm{m}}\right)-\left(\varepsilon_{\mathrm{i}}-\varepsilon_{\mathrm{m}}\right) f\right]^{2}} .
\end{aligned}
$$

However, to evaluate the remaining three derivatives in Eq. (10), we need to consider the mechanical response of the metamaterial to the induced pressure field (3).

Since shear stress is omitted in our model, the matrix and inclusions do not undergo deformations, but instead experience a compression in order to preserve hydrostatic equilibrium. This is seen mathematically by stating that perturbations to the material pressure fields remain continuous across the boundary of the sphere

$$
\left.\Delta P_{\mathrm{i}}\right|_{\partial \Omega}=\left.\Delta P_{\mathrm{m}}\right|_{\partial \Omega},
$$

where $P_{\mathrm{i}, \mathrm{m}}$ denotes the pressure fields and $\partial \Omega$ is the boundary of the inclusion. We can then evaluate Taylor series for the constituent volumes $V_{\mathrm{i}, \mathrm{m}}$ with respect to $P_{\mathrm{i}, \mathrm{m}}$ to obtain

$$
\begin{aligned}
\Delta V_{\mathrm{i}} & =\frac{\partial V_{\mathrm{i}}}{\partial P_{\mathrm{i}}} \Delta P_{\mathrm{i}}, \\
\Delta V_{\mathrm{m}} & =\frac{\partial V_{\mathrm{m}}}{\partial P_{\mathrm{m}}} \Delta P_{\mathrm{m}},
\end{aligned}
$$

and express (12) in the form

$$
\left.\frac{\Delta V_{\mathrm{i}}}{V_{\mathrm{i}} \beta_{\mathrm{i}}}\right|_{\partial \Omega}=\left.\frac{\Delta V_{\mathrm{m}}}{V_{\mathrm{m}} \beta_{\mathrm{m}}}\right|_{\partial \Omega},
$$

where we have introduced the compressibility constant

$$
\beta=-\frac{1}{V} \frac{\partial V}{\partial P}=K^{-1},
$$

and $K$ denotes the bulk modulus. Integrating both sides of (14) we obtain the interface condition

$$
V_{\mathrm{m}}=A\left[V_{\mathrm{i}}\right]^{\beta_{\mathrm{m}} / \beta_{\mathrm{i}}},
$$

for some constant $A$, which describes the compressive response of our composite. With this condition, and using the definition for the composite density

$$
\rho=\rho_{\mathrm{i}} f+\rho_{\mathrm{m}}(1-f)=\frac{m_{\mathrm{i}}+m_{\mathrm{m}}}{V_{\mathrm{i}}+V_{\mathrm{m}}},
$$

we evaluate the remaining three derivatives to obtain

$$
\begin{aligned}
\frac{\partial \varepsilon_{\mathrm{m}}}{\partial \rho} & =\frac{\partial \varepsilon_{\mathrm{m}}}{\partial \rho_{\mathrm{m}}} \frac{\partial \rho_{\mathrm{m}}}{\partial V_{\mathrm{i}}} \frac{\partial V_{\mathrm{i}}}{\partial \rho}=\frac{\gamma_{\mathrm{m}}}{\rho} \frac{\beta_{\mathrm{m}}}{\beta_{\mathrm{c}}}, \\
\frac{\partial \varepsilon_{\mathrm{i}}}{\partial \rho} & =\frac{\partial \varepsilon_{\mathrm{i}}}{\partial \rho_{\mathrm{i}}} \frac{\partial \rho_{\mathrm{i}}}{\partial V_{\mathrm{i}}} \frac{\partial V_{\mathrm{i}}}{\partial \rho}=\frac{\gamma_{\mathrm{i}}}{\rho} \frac{\beta_{\mathrm{i}}}{\beta_{\mathrm{c}}}, \\
\frac{\partial f}{\partial \rho} & =\frac{\partial f}{\partial V_{\mathrm{i}}} \frac{\partial V_{\mathrm{i}}}{\partial \rho}=\frac{f(1-f)}{\rho} \frac{\left(\beta_{\mathrm{m}}-\beta_{\mathrm{i}}\right)}{\beta_{\mathrm{c}}},
\end{aligned}
$$

where analogously to (5a) we introduce $\gamma_{\mathrm{m}}=\rho_{\mathrm{m}} \partial \varepsilon_{\mathrm{m}} / \partial \rho_{\mathrm{m}}$ and $\gamma_{\mathrm{i}}=\rho_{\mathrm{i}} \partial \varepsilon_{\mathrm{i}} / \partial \rho_{\mathrm{i}}$ as the electrostriction values of the constituent media, and $\beta_{\mathrm{c}}=\beta_{\mathrm{i}} f+\beta_{\mathrm{m}}(1-f)$ denotes the volume-averaged compressibility over the unit cell. Consequently, the nondispersive electrostriction for our composite is given by

$$
\begin{aligned}
\gamma^{\mathrm{ND}}= & \frac{\beta_{\mathrm{i}} f}{\beta_{\mathrm{c}}}\left[\frac{\left(\varepsilon_{\mathrm{i}}+2 \varepsilon_{\mathrm{m}}\right)-\left(\varepsilon_{\mathrm{i}}-\varepsilon_{\mathrm{m}}\right)}{\left(\varepsilon_{\mathrm{i}}+2 \varepsilon_{\mathrm{m}}\right)-\left(\varepsilon_{\mathrm{i}}-\varepsilon_{\mathrm{m}}\right) f}\right]^{2} \gamma_{\mathrm{i}} \\
& +\frac{\beta_{\mathrm{m}}(1-f)}{\beta_{\mathrm{c}}}\left[\frac{\left(\varepsilon_{\mathrm{i}}+2 \varepsilon_{\mathrm{m}}\right)^{2}+2 f\left(\varepsilon_{\mathrm{i}}-\varepsilon_{\mathrm{m}}\right)^{2}}{\left[\left(\varepsilon_{\mathrm{i}}+2 \varepsilon_{\mathrm{m}}\right)-\left(\varepsilon_{\mathrm{i}}-\varepsilon_{\mathrm{m}}\right) f\right]^{2}}\right] \gamma_{\mathrm{m}} \\
& +\underbrace{\frac{\left(\beta_{\mathrm{m}}-\beta_{\mathrm{i}}\right) f(1-f)}{\beta_{\mathrm{c}}}\left[\frac{3 \varepsilon_{\mathrm{m}}\left(\varepsilon_{\mathrm{i}}-\varepsilon_{\mathrm{m}}\right)\left(\varepsilon_{\mathrm{i}}+2 \varepsilon_{\mathrm{m}}\right)}{\left[\left(\varepsilon_{\mathrm{i}}+2 \varepsilon_{\mathrm{m}}\right)-\left(\varepsilon_{\mathrm{i}}-\varepsilon_{\mathrm{m}}\right) f\right]^{2}}\right]}_{\text {artificial electrostriction }},
\end{aligned}
$$

which is a weighted linear function of the constituent electrostriction values $\gamma_{\mathrm{i}}$ and $\gamma_{\mathrm{m}}$ plus a new artificial electrostriction term (highlighted). The latter term can be understood by considering the limit $\gamma_{\mathrm{i}}=\gamma_{\mathrm{m}}=0$; if the two materials have different compressibility values, then compression leads to a change in the filling fraction $f$, which, if $\varepsilon_{\mathrm{i}} \neq \varepsilon_{\mathrm{m}}$, alters the effective dielectric constant (8). Another interesting feature of (19) is the second-order pole present in all terms at

$$
f=\left(\varepsilon_{\mathrm{i}}+2 \varepsilon_{\mathrm{m}}\right) /\left(\varepsilon_{\mathrm{i}}-\varepsilon_{\mathrm{m}}\right),
$$

giving a theoretically infinite value for the composite electrostriction. However, this resonance can only be obtained with a change in sign for either $\varepsilon_{\mathrm{i}}$ or $\varepsilon_{\mathrm{m}}$ for dilute, positive $f$. A discussion of the asymptotic behavior of (19) with respect to $\beta_{\mathrm{i}, \mathrm{m}}$ and $\varepsilon_{\mathrm{i}, \mathrm{m}}$ is presented in the Appendix for completeness.

Next, we consider the composite $\gamma$ expression when dispersion is included.

\section{B. Dispersive corrections}

In this section, we incorporate dispersion in the derivation of the composite $\gamma$. We begin by returning to (4), which from the MG model (8) has the form

$$
\begin{aligned}
\gamma= & \rho\left[\frac{\partial \varepsilon_{\mathrm{r}}}{\partial \varepsilon_{\mathrm{m}}} \frac{\partial \varepsilon_{\mathrm{m}}}{\partial \rho}+\frac{\partial \varepsilon_{\mathrm{r}}}{\partial \varepsilon_{\mathrm{i}}} \frac{\partial \varepsilon_{\mathrm{i}}}{\partial \rho}+\frac{\partial \varepsilon_{\mathrm{r}}}{\partial f} \frac{\partial f}{\partial \rho}\right] \\
& +\rho \omega \frac{\partial}{\partial \rho}\left\{\frac{\partial \varepsilon_{\mathrm{r}}}{\partial \varepsilon_{\mathrm{m}}} \frac{\partial \varepsilon_{\mathrm{m}}}{\partial \omega}+\frac{\partial \varepsilon_{\mathrm{r}}}{\partial \varepsilon_{\mathrm{i}}} \frac{\partial \varepsilon_{\mathrm{i}}}{\partial \omega}+\frac{\partial \varepsilon_{\mathrm{r}}}{\partial f} \frac{\partial f}{\partial \omega}\right\} .
\end{aligned}
$$

This composite expression is then decomposed in the form $\gamma=$ $\gamma^{\mathrm{ND}}+\gamma^{\mathrm{D}}$, where $\gamma^{\mathrm{ND}}$ and $\gamma^{\mathrm{D}}$ represent the nondispersive and dispersive contributions, respectively. The nondispersive contribution is given by the first three terms of (21) and has 
been evaluated in the previous section as (19), where we introduce the substitution $\gamma_{\mathrm{i}, \mathrm{m}}=\gamma_{\mathrm{i}, \mathrm{m}}^{\mathrm{ND}}$ therein.

Next, we evaluate the remaining terms in Eq. (21), and note that we have

$$
\frac{\partial f}{\partial \omega}=0,
$$

as all mechanical parameters, such as $\beta$ and $\rho$, are independent of the optical frequency. Accordingly, we decompose the dispersive term $\gamma^{\mathrm{D}}$ into a matrix and inclusion contribution $\gamma^{\mathrm{D}}=\gamma_{\mathcal{M}}^{\mathrm{D}}+\gamma_{\mathcal{I}}^{\mathrm{D}}$ and obtain

$$
\begin{aligned}
\gamma_{\mathcal{M}}^{\mathrm{D}}= & \rho \omega \frac{\partial \varepsilon_{\mathrm{m}}}{\partial \omega} \frac{3}{\left[\left(\varepsilon_{\mathrm{i}}+2 \varepsilon_{\mathrm{m}}\right)-\left(\varepsilon_{\mathrm{i}}-\varepsilon_{\mathrm{m}}\right) f\right]^{3}} \\
& \times\left[\frac{\partial \varepsilon_{\mathrm{i}}}{\partial \rho}\left\{6 \varepsilon_{\mathrm{i}} \varepsilon_{\mathrm{m}} f(1-f)\right\}-\frac{\partial \varepsilon_{\mathrm{m}}}{\partial \rho}\left\{6 \varepsilon_{\mathrm{i}}^{2} f(1-f)\right\}\right. \\
& +\frac{\partial f}{\partial \rho}\left\{\varepsilon_{\mathrm{i}}^{3}(1-f)-3 \varepsilon_{\mathrm{i}}^{2} \varepsilon_{\mathrm{m}} f-2 \varepsilon_{\mathrm{m}}^{3}(f+2)\right. \\
& \left.\left.-6 \varepsilon_{\mathrm{i}} \varepsilon_{\mathrm{m}}^{2}(1-f)\right\}\right] \\
& +\frac{\beta_{\mathrm{m}}(1-f)}{\beta_{\mathrm{c}}}\left[\frac{\left(\varepsilon_{\mathrm{i}}+2 \varepsilon_{\mathrm{m}}\right)^{2}+2 f\left(\varepsilon_{\mathrm{i}}-\varepsilon_{\mathrm{m}}\right)^{2}}{\left[\left(\varepsilon_{\mathrm{i}}+2 \varepsilon_{\mathrm{m}}\right)-f\left(\varepsilon_{\mathrm{i}}-\varepsilon_{\mathrm{m}}\right)\right]^{2}}\right] \gamma_{\mathrm{m}}^{D}
\end{aligned}
$$

and

$$
\begin{aligned}
\gamma_{\mathcal{I}}^{\mathrm{D}}= & \rho \omega \frac{\partial \varepsilon_{\mathrm{i}}}{\partial \omega} \frac{9 \varepsilon_{\mathrm{m}}}{\left[\left(\varepsilon_{\mathrm{i}}+2 \varepsilon_{\mathrm{m}}\right)-\left(\varepsilon_{\mathrm{i}}-\varepsilon_{\mathrm{m}}\right) f\right]^{3}} \\
& \times\left[\frac{\partial \varepsilon_{\mathrm{m}}}{\partial \rho}\left\{2 \varepsilon_{\mathrm{i}} f(1-f)\right\}-\frac{\partial \varepsilon_{\mathrm{i}}}{\partial \rho}\left\{2 \varepsilon_{\mathrm{m}} f(1-f)\right\}\right. \\
& \left.+\frac{\partial f}{\partial \rho}\left\{\varepsilon_{\mathrm{m}}\left[\left(\varepsilon_{\mathrm{i}}+2 \varepsilon_{\mathrm{m}}\right)+f\left(\varepsilon_{\mathrm{i}}-\varepsilon_{\mathrm{m}}\right)\right]\right\}\right] \\
& +\frac{\beta_{\mathrm{i}} f}{\beta_{\mathrm{c}}}\left[\frac{\left(\varepsilon_{\mathrm{i}}+2 \varepsilon_{\mathrm{m}}\right)-\left(\varepsilon_{\mathrm{i}}-\varepsilon_{\mathrm{m}}\right)}{\left(\varepsilon_{\mathrm{i}}+2 \varepsilon_{\mathrm{m}}\right)-f\left(\varepsilon_{\mathrm{i}}-\varepsilon_{\mathrm{m}}\right)}\right]^{2} \gamma_{\mathrm{i}}^{D} .
\end{aligned}
$$

The remaining derivatives with respect to $\rho$ in Eqs. (23) and (24) are given in Eq. (18) where we use the substitutions $\gamma_{\mathrm{i}, \mathrm{m}}=\gamma_{\mathrm{i}, \mathrm{m}}^{\mathrm{ND}}$ therein.

In summary, the expression for the dispersive composite electrostriction is given by

$$
\gamma=\gamma^{\mathrm{ND}}+\gamma_{\mathcal{M}}^{\mathrm{D}}+\gamma_{\mathcal{I}}^{\mathrm{D}}
$$

where $\gamma_{\mathrm{i}, \mathrm{m}}=\gamma_{\mathrm{i}, \mathrm{m}}^{\mathrm{ND}}$ has been substituted appropriately. Using the full definitions (4) for the constituents $\gamma_{i, m}$, (25) has an identical structure to that presented for the nondispersive expression in Eq. (19), except now the artificial electrostriction term is modified by additional terms. These dispersive contributions (23) and (24) feature the same MG resonance (20) as before, but with a contribution from a third-order pole. This suggests that the omission of dispersion can, in certain instances, have considerable influence on the result for composite $\gamma$.

\section{NUMERICAL EXAMPLES}

In this section, we investigate the composite electrostriction expressions (19) and (25) for our structure, using combinations (a)



(b)

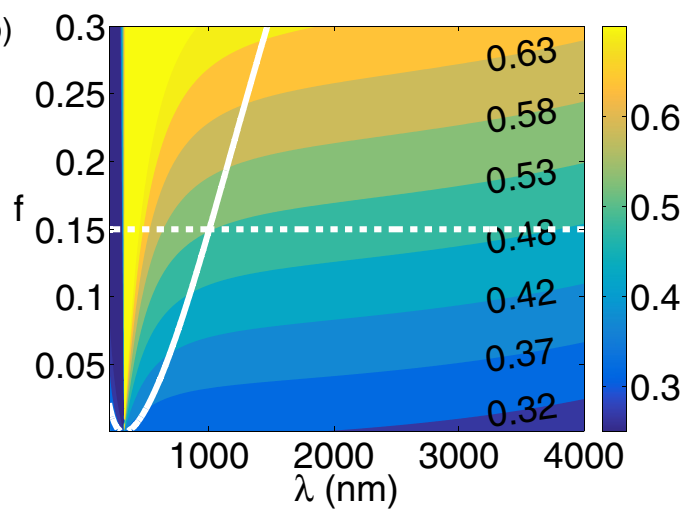

FIG. 2. (Color online) Contour plots of (a) $\log _{10}|\operatorname{Re}(\gamma)|$ from (25), and (b) $\log _{10}\left|\operatorname{Re}\left(\varepsilon_{\mathrm{r}}\right)\right|$ from (8), for an array of $\mathrm{Ag}$ spheres embedded in $\mathrm{SiO}_{2}$ matrix, against filling fraction $f$ and wavelength $\lambda$. A diluteness threshold of $f=15 \%$ (dashed white curve) and an attenuation length threshold of $\alpha_{\mathrm{L}}=0.1 \mathrm{~mm}$ (solid white curve) are also shown.

of different materials. We accompany this investigation with an analysis of the losses for these designs, which is necessary for realistic applications. Accordingly, we return to the MG model (8) and define the attenuation length

$$
\alpha_{\mathrm{L}}=\left[\frac{4 \pi}{\lambda} \operatorname{Im}\left(\sqrt{\varepsilon_{\mathrm{r}}}\right)\right]^{-1},
$$

which we emphasize is completely independent from the electrostriction analysis. From (26), a threshold of $\alpha_{\mathrm{L}} \geqslant$ $0.1 \mathrm{~mm}$ is imposed as a tolerance for omitting dissipation effects, which is also a typical interaction length for SBS.

We begin by investigating the composite electrostriction (25) for a cubic array of silver [24] spheres embedded in a silica [25] matrix, where we use (7) for $\gamma_{i}$. In Fig. 2(a), we present a contour plot of $\log _{10}|\operatorname{Re}(\gamma)|$ over the wavelength range $350 \mathrm{~nm} \leqslant \lambda \leqslant 4000 \mathrm{~nm}$ for filling fraction $0 \leqslant f \leqslant$ 0.3 .

A striking feature of this figure is the region corresponding to $\log _{10}|\operatorname{Re}(\gamma)|>0.8$, which contains the permittivity resonance (20). This region simply denotes $\gamma$ values over a cutoff threshold, which is introduced to ensure that features of the contour plot are not dominated by the singularity in Eq. (25). We note that the extremely strong enhancements in $\gamma$ courtesy of (20) are associated with strong attenuation (26), and we highlight this by superposing a solid white curve over these contours, which represents an attenuation length threshold of 

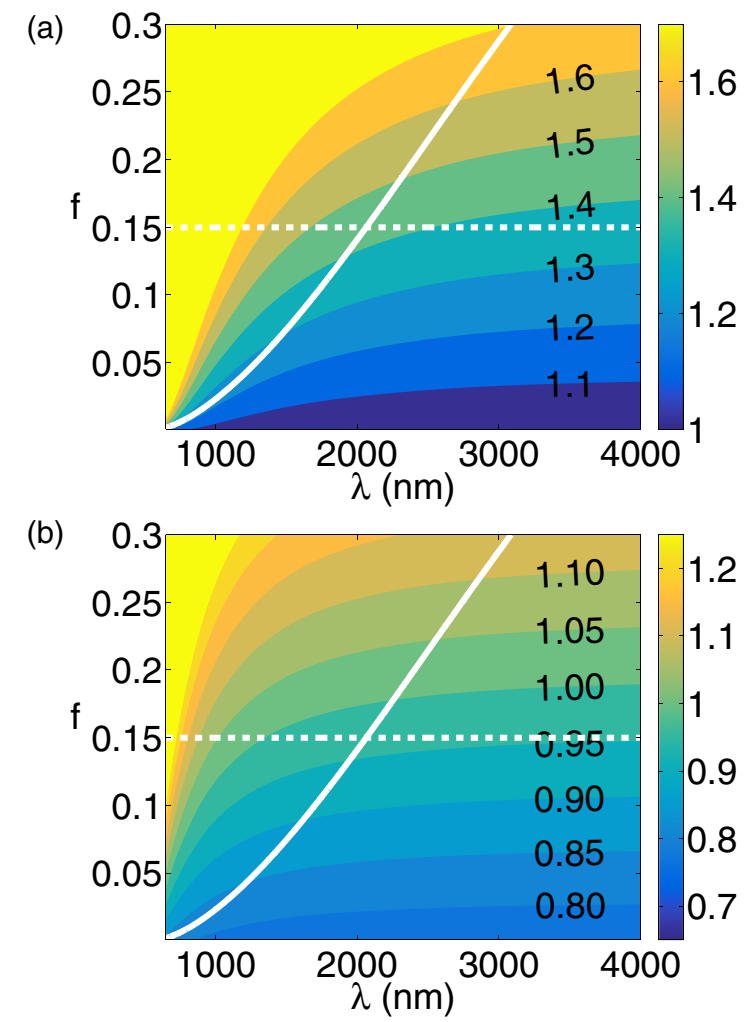

FIG. 3. (Color online) Contour plots of (a) $\log _{10}|\operatorname{Re}(\gamma)|$ from (25), and (b) $\log _{10}\left|\operatorname{Re}\left(\varepsilon_{\mathrm{r}}\right)\right|$ from (8), for an array of $\mathrm{Ag}$ spheres embedded in a $\mathrm{As}_{2} \mathrm{~S}_{3}$ matrix, against filling fraction $f$ and wavelength $\lambda$. A diluteness threshold of $f=15 \%$ (dashed white curve) and an attenuation length threshold of $\alpha_{\mathrm{L}}=0.1 \mathrm{~mm}$ (solid white curve) are also shown.

$\alpha_{\mathrm{L}}=0.1 \mathrm{~mm}$ (where to the right of this curve we have longer $\alpha_{\mathrm{L}}$, and to the left, a region of shorter lengths).

Also shown is a dashed white curve, which represents our diluteness threshold of $f=15 \%$. Accordingly, inside the region bound by these two curves [the region of validity (ROV)], we find a maximum composite electrostriction value of $\gamma=3.27$ at $(\lambda, f)=(1003 \mathrm{~nm}, 0.15)$, which corresponds to the intersection of the $\alpha_{\mathrm{L}}$ and $f$ curves. This point gives an enhancement factor of 3.36 relative to the electrostriction for the silica background at the same wavelength. It is also clear from these contours that the electrostriction is tunable over a wide wavelength interval, but that these enhancements are ultimately constrained by the diluteness requirement of the MG model.

In Fig. 2(b), we present a contour plot of the effective permittivity over the same $(\lambda, f)$ range, where the plasmon resonance is clearly visible. For our maximum electrostriction value at $(\lambda, f)=(1003 \mathrm{~nm}, 0.15)$, we have a composite permittivity of $\varepsilon_{\mathrm{r}}=3.4+0.003 \mathrm{i}$, which is an enhancement factor of 1.6 relative to the background value of $\varepsilon_{\mathrm{m}}=2.10$ at the same wavelength. As one would expect, this contour plot features similar curvature to that of $\gamma$, and a low degree of frequency dependence within the ROV $\left[1.9<\operatorname{Re}\left(\varepsilon_{\mathrm{r}}\right)<3.4\right]$.

We now consider silver spheres embedded in a chalcogenide [26] matrix (amorphous $\mathrm{As}_{2} \mathrm{~S}_{3}$ ). In Fig. 3(a) we present the composite $\gamma$ for this configuration, and observe (a)

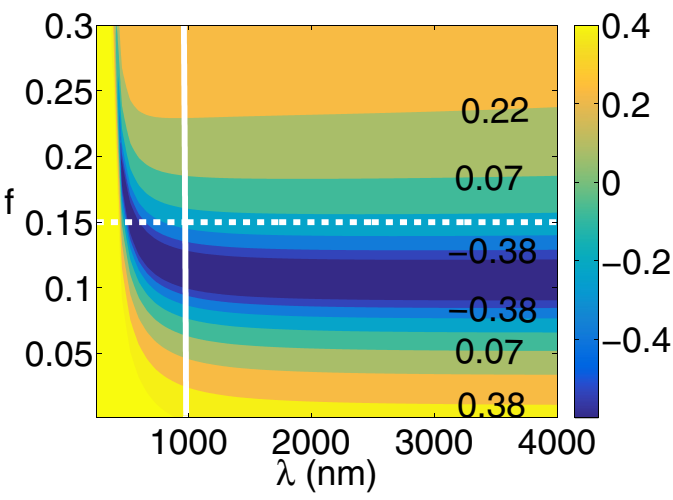

(b)

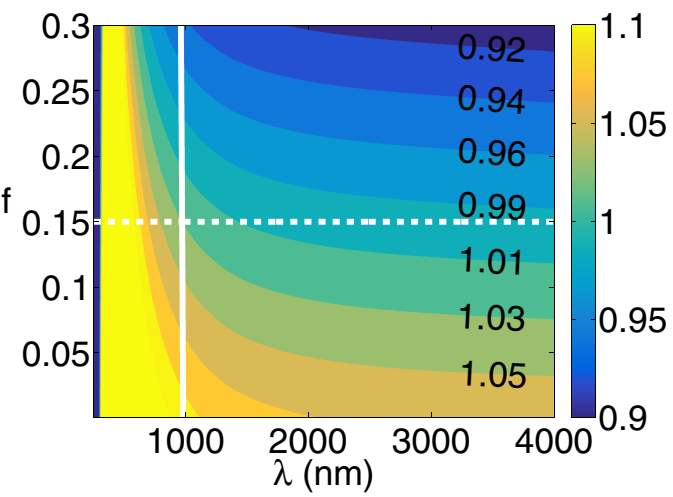

FIG. 4. (Color online) Contour plots of (a) $\log _{10}|\operatorname{Re}(\gamma)|$ from (19), and (b) $\log _{10}\left|\operatorname{Re}\left(\varepsilon_{\mathrm{r}}\right)\right|$ from (8), for an array of $\mathrm{SiO}_{2}$ spheres embedded in a Si matrix, against filling fraction $f$ and wavelength $\lambda$. A diluteness threshold of $f=15 \%$ (dashed white curve) and an attenuation length threshold of $\alpha_{\mathrm{L}}=0.1 \mathrm{~mm}$ (solid white curve) are also shown.

qualitatively similar behavior to the previous example for a silica matrix in Fig. 3(a). The primary difference here is the much more restrictive $\alpha_{\mathrm{L}}$ threshold, which now extends to much longer wavelengths.

If one searches inside the ROV constrained by our $\alpha_{\mathrm{L}}$ and $f$ bounds, we discover a maximum electrostriction value of $\gamma=27.4$ at $(\lambda, f)=(2064 \mathrm{~nm}, 0.15)$, corresponding to the intersection of the $\alpha_{\mathrm{L}}$ and $f$ curves as before, with an enhancement factor of 2.63 (cf. $\gamma_{\mathrm{m}}=10.44$ ). Figure 3(b) reveals that this coordinate point has an effective permittivity value of $\varepsilon_{\mathrm{r}}=9.3+0.01 \mathrm{i}$. This corresponds to a similar permittivity enhancement factor as the previous example (cf. $\varepsilon_{\mathrm{m}}=5.89$ ). A slightly higher level of frequency dependence is observed in the $\operatorname{ROV}$ also $\left[5.8<\operatorname{Re}\left(\varepsilon_{\mathrm{r}}\right)<9.3\right]$.

For these examples, we find that the composite electrostriction expression (25) gives a 10\%-20\% increase in the maximum electrostriction value compared to the nondispersive expression (19). This suggests that the omission of dispersion can give rise to a small but non-negligible correction to the composite electrostriction. Furthermore, a similar investigation with $\mathrm{Au}$ spheres embedded in these matrix materials reveals a comparable level of enhancement to $\mathrm{Ag}$.

In Fig. 4(a), we consider $\log _{10}|\operatorname{Re}(\gamma)|$ from (19) for silica spheres embedded in a silicon [27] matrix. This figure exhibits strong frequency dependence for $\lambda<1000 \mathrm{~nm}$ (courtesy of a material resonance for $\mathrm{Si}$ at $\lambda \approx 370 \mathrm{~nm}$ ) and a near-horizontal arc of zero electrostriction which spans the entire ROV. That is, 
(a)

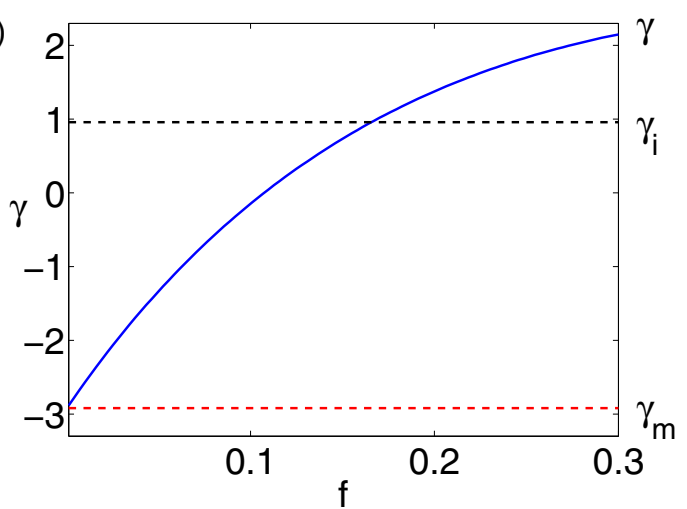

(b)

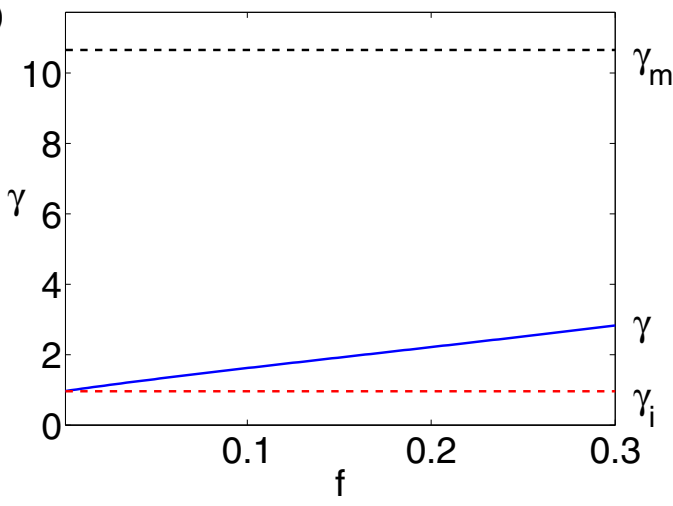

FIG. 5. (Color online) Composite electrostriction $\operatorname{Re}(\gamma)$ from (19) at $\lambda=1550 \mathrm{~nm}$ for an array of (a) $\mathrm{SiO}_{2}$ spheres embedded in a $\mathrm{Si}$ matrix, and (b) $\mathrm{As}_{2} \mathrm{~S}_{3}$ spheres embedded in $\mathrm{SiO}_{2}$ matrix. The electrostriction for the inclusion $\gamma_{\mathrm{i}}$ (dashed black line) and matrix $\gamma_{\mathrm{m}}$ (dashed red line) materials are also given.

this metamaterial design can completely suppress electrostriction over an exceptionally wide frequency range. For this particular composite, the attenuation length threshold is reached at approximately $\lambda=1000 \mathrm{~nm}$. In Fig. 4(b), we present a contour plot of $\log _{10}\left|\operatorname{Re}\left(\epsilon_{r}\right)\right|$ for completeness, which exhibits reassuringly minimal frequency dependence over the ROV.

In Fig. 5(a) we show a cross section of the composite $\operatorname{Re}(\gamma)$ from Fig. 4(a) at $\lambda=1550 \mathrm{~nm}$. This gives confirmation that complete suppression of electrostriction is achieved at $f \approx$ $10 \%$, and shows that we have sign-changing electrostriction from this metamaterial design. We note that the composite $\operatorname{Re}(\gamma)$ (blue curve) exceeds that of the constituent electrostriction values (dashed curves) at a filling fraction of $f=16.6 \%$, which is reminiscent of earlier work which showed the nonlinear parameters of composite materials can exceed the values of the constituents [10], but we note that care must be taken as the dilute lattice assumption breaks down in this region of enhancement. An investigation using $\mathrm{As}_{2} \mathrm{~S}_{3}$ spheres in an Si matrix demonstrated an identical result to that shown in Figs. 4(a), 4(b), and 5(a), but at much lower filling fractions.

To emphasize the result presented in Fig. 5(a), we show the composite $\gamma$ curve for an array of chalcogenide $\left(\mathrm{As}_{2} \mathrm{~S}_{3}\right)$ spheres embedded in a silica matrix at $\lambda=1550 \mathrm{~nm}$ in Fig. 5(b). This shows a simple linear enhancement from the background electrostriction, to a maximum realizable value of $\gamma=1.918$ at the threshold of $f=15 \%$ (i.e., an enhancement factor of approximately 2 ).

\section{CONCLUDING REMARKS}

We have presented an analytical representation for the electrostriction of a composite material by incorporating the simplest and analytically most transparent model from effective index theory, the Maxwell-Garnett model, to the problem of electrostriction.

We show that expressions for the electrostriction of a composite material feature artificial electrostriction terms, which contribute to the enhancement or suppression of this material property, as observed for a selection of composites here. The presence of this term points towards the possibility that large enhancements in $\gamma$, beyond both material values, could be achieved for more sophisticated metamaterial designs. We also show that sign-switching electrostriction is achievable, and that resonant enhancements in the electrostriction of metaldielectric composites are unrealistic, as they are associated with strong attenuation. Incorporating dispersive effects in the model is shown to give a small but non-negligible correction to estimates for the composite electrostriction.

It is important to emphasize that this work is a first step in the study of the electrostriction of composites, and so other considerations such as thermally induced electrostriction and scattering losses are not addressed here. These effects are more prominent for high-intensity wave problems, where more elaborate models are required to accurately evaluate the material response. Including the effect of shear stresses will require a fully tensorial description of all stress fields, and is the next step in the development of the theory. Also, we note that our estimate for the electrostriction of metals is a low-order approximation, which requires experimental data for validation.

As a final comment, we emphasize that other homogenization procedures [23] can be used to determine the electrostriction for a periodic composite, which should remove several constraints of the present MG model, and open the way to investigations of exciting metamaterial designs.

Note added in proof. Recently, we became aware of a preprint [28] on a similar topic.

\section{ACKNOWLEDGMENTS}

This work was supported by the Australian Research Council (CUDOS Centre of Excellence, CE110001018).

\section{APPENDIX: ASYMPTOTIC ANALYSIS OF THE NONDISPERSIVE COMPOSITE ELECTROSTRICTION}

In this Appendix, we examine several asymptotic limits for the composite electrostriction expression (19). First, assuming $\varepsilon_{\mathrm{m}} \gg \varepsilon_{\mathrm{i}}$, we obtain

$\gamma \sim \frac{9 \beta_{\mathrm{i}} f}{\beta_{\mathrm{c}}(f+2)^{2}} \gamma_{\mathrm{i}}-\frac{2 \beta_{\mathrm{m}}(f-1)}{\beta_{\mathrm{c}}(f+2)} \gamma_{\mathrm{m}}+\frac{6\left(\beta_{\mathrm{i}}-\beta_{\mathrm{m}}\right) f(1-f) \varepsilon_{\mathrm{m}}}{\beta_{\mathrm{c}}(f+2)^{2}}$,

where we have a persistent, but simplified, contribution from all terms in Eq. (19). By contrast, for $\varepsilon_{\mathrm{i}} \gg \varepsilon_{\mathrm{m}}$ we have

$\gamma \sim \frac{9 f \beta_{\mathrm{i}}}{(1-f)^{2} \beta_{\mathrm{c}}}\left(\frac{\varepsilon_{\mathrm{m}}^{2}}{\varepsilon_{\mathrm{i}}^{2}}\right) \gamma_{\mathrm{i}}+\frac{\beta_{\mathrm{m}}(1+2 f)}{(1-f) \beta_{\mathrm{c}}} \gamma_{\mathrm{m}}+\frac{3 \varepsilon_{\mathrm{m}} f\left(\beta_{\mathrm{m}}-\beta_{\mathrm{i}}\right)}{(1-f) \beta_{\mathrm{c}}}$. 
The limit $\beta_{\mathrm{m}} \gg \beta_{\mathrm{i}}$ gives the asymptotic form

$$
\gamma \sim\left[\frac{\left(\varepsilon_{\mathrm{i}}+2 \varepsilon_{\mathrm{m}}\right)^{2}+2 f\left(\varepsilon_{\mathrm{i}}-\varepsilon_{\mathrm{m}}\right)^{2}}{\left[\left(\varepsilon_{\mathrm{i}}+2 \varepsilon_{\mathrm{m}}\right)-\left(\varepsilon_{\mathrm{i}}-\varepsilon_{\mathrm{m}}\right) f\right]^{2}}\right] \gamma_{\mathrm{m}}+\frac{3 f \varepsilon_{\mathrm{m}}\left(\varepsilon_{\mathrm{i}}-\varepsilon_{\mathrm{m}}\right)\left(\varepsilon_{\mathrm{i}}+2 \varepsilon_{\mathrm{m}}\right)}{\left[\left(\varepsilon_{\mathrm{i}}+2 \varepsilon_{\mathrm{m}}\right)-\left(\varepsilon_{\mathrm{i}}-\varepsilon_{\mathrm{m}}\right) f\right]^{2}},
$$

and $\beta_{\mathrm{i}} \gg \beta_{\mathrm{m}}$ leads to

$$
\gamma \sim\left[\frac{\left(\varepsilon_{\mathrm{i}}+2 \varepsilon_{\mathrm{m}}\right)-\left(\varepsilon_{\mathrm{i}}-\varepsilon_{\mathrm{m}}\right)}{\left(\varepsilon_{\mathrm{i}}+2 \varepsilon_{\mathrm{m}}\right)-\left(\varepsilon_{\mathrm{i}}-\varepsilon_{\mathrm{m}}\right) f}\right]^{2} \gamma_{\mathrm{i}}-\frac{3(1-f) \varepsilon_{\mathrm{m}}\left(\varepsilon_{\mathrm{i}}-\varepsilon_{\mathrm{m}}\right)\left(\varepsilon_{\mathrm{i}}+2 \varepsilon_{\mathrm{m}}\right)}{\left[\left(\varepsilon_{\mathrm{i}}+2 \varepsilon_{\mathrm{m}}\right)-\left(\varepsilon_{\mathrm{i}}-\varepsilon_{\mathrm{m}}\right) f\right]^{2}} .
$$

These differences in sign in the artificial electrostriction contributions above suggest that the relative magnitudes of $\beta$ are relevant in establishing whether enhanced or suppressed electrostriction is observed.

[1] B. J. Eggleton, C. G. Poulton, and R. Pant, Adv. Opt. Photonics 5, 536 (2013).

[2] L. Brillouin, Ann. Phys. (Paris) 17, 88 (1922).

[3] L. Mandelstam, Zh. Russ. Fiz-Khim. Ova. 58, 381 (1926).

[4] R. Y. Chiao, E. Garmire, and C. H. Townes, Phys. Rev. Lett. 13, 479 (1964).

[5] M. Lapine, I. V. Shadrivov, and Y. S. Kivshar, Rev. Mod. Phys. 86, 1093 (2014).

[6] V. M. Agranovich, Y. R. Shen, R. H. Baughman, and A. A. Zakhidov, Phys. Rev. B 69, 165112 (2004).

[7] N. Segal, S. Keren-Zur, N. Hendler, and T. Ellenbogen, Nat. Photonics 9, 180 (2015).

[8] M. Aspelmeyer, T. J. Kippenberg, and F. Marquardt, Rev. Mod. Phys. 86, 1391 (2014).

[9] M. Lapine, I. V. Shadrivov, D. A. Powell, and Y. S. Kivshar, Nat. Mater. 11, 30 (2012).

[10] J. E. Sipe and R. W. Boyd, Phys. Rev. A 46, 1614 (1992).

[11] R. W. Boyd, Nonlinear Optics, 3rd ed. (Academic, London, 2003).

[12] I. L. Fabelinskii, Molecular Scattering of Light (Springer, New York, 1968).

[13] L. D. Landau, E. M. Lifshitz, and L. P. Pitaevskii, Electrodynamics of Continuous Media, 2nd ed. (Pergamon, Oxford, 1984), Vol. 8.
[14] P. T. Rakich, P. Davids, and Z. Wang, Opt. Express 18, 14439 (2010).

[15] J. A. Stratton, Electromagnetic Theory (Wiley, Hoboken, 2007).

[16] D. F. Nelson and M. Lax, Phys. Rev. B 3, 2778 (1971).

[17] H. Mueller, Phys. Rev. 47, 947 (1935).

[18] A. Melloni, M. Frasca, A. Garavaglia, A. Tonini, and M. Martinelli, Opt. Lett. 23, 691 (1998).

[19] N. A. Stakhin, Russ. Phys. J. 41, 1107 (1998).

[20] M. J. Weber, Handbook of Optical Materials (CRC Press, Boca Raton, 2002).

[21] D. K. Biegelsen, Phys. Rev. Lett. 32, 1196 (1974).

[22] C. F. Bohren and D. R. Huffman, Absorption and Scattering of Light by Small Particles (Wiley, New York, 2008).

[23] G. W. Milton, The Theory of Composites (Cambridge University Press, New York, 2002).

[24] M. A. Ordal, R. J. Bell, R. W. Alexander Jr., L. L. Long, and M. R. Querry, Appl. Opt. 24, 4493 (1985).

[25] I. H. Malitson, J. Opt. Soc. Am. 55, 1205 (1965).

[26] Amtir-6 information, http://www.amorphousmaterials.com/app/ download/6552919404/AMTIR-6+Information.pdf.

[27] M. A. Green, Sol. Energy Mater. Sol. Cells 92, 1305 (2008).

[28] W. Sun, S. B. Wang, J. Ng, L. Zhou, and C. T. Chan, arXiv:1504.06437. 